\title{
Lock-in thermography as a rapid and reproducible thermal characterization method for magnetic nanoparticles
}

Philipp Lemal ${ }^{\mathrm{a}, \uparrow}$, Christoph Geers ${ }^{\mathrm{a}, \uparrow}$, Christophe A. Monnier ${ }^{\mathrm{a}}$, Federica Crippa ${ }^{\mathrm{a}}$, Leopold Daum ${ }^{\mathrm{a}}$, Dominic A. Urban ${ }^{\mathrm{a}}$, Barbara Rothen-Rutishauser ${ }^{\mathrm{a}}$, Mathias Bonmarin ${ }^{\mathrm{b}} *$, Alke Petri-Fink ${ }^{\mathrm{a}, \mathrm{c}, *}$, Thomas L. Moore ${ }^{\mathrm{a}, *}$

${ }^{a}$ Adolphe Merkle Institute, University of Fribourg, Chemin des Verdiers 4, CH-1700 Fribourg, Switzerland

${ }^{b}$ Institute of Computational Physics, Zurich University of Applied Sciences, Technikumstrasse 9, 8400 Winterthur, Switzerland

${ }^{c}$ Chemistry Department, University of Fribourg, Chemin du Museé 9, CH-1700 Fribourg Switzerland

* Corresponding authors: thomaslee.moore@unifr.ch, mathias.bonmarin@ zhaw.ch, alke.fink@unifr.ch

${ }^{\dagger}$ These authors contributed equally to this manuscript

Keywords: lock-in thermography, magnetic nanoparticles, hyperthermia, nanoparticle characterization.

\begin{abstract}
Lock-in thermography (LIT) is a sensitive imaging technique generally used in engineering and materials science (e.g. detecting defects in composite materials). However, it has recently been expanded for investigating the heating power of nanomaterials, such as superparamagnetic iron oxide nanoparticles (SPIONs). Here we implement LIT as a rapid and reproducible method that can evaluate the heating potential of various sizes of SPIONs under an alternating magnetic field (AMF), as well as the limits of detection for each particle size. SPIONs were synthesized via thermal decomposition and stabilized in water via a ligand transfer process. Thermographic measurements of SPIONs were made by stimulating particles of varying sizes and increasing concentrations under an AMF. Furthermore, a commercially available SPION sample was included as an external reference. While the size dependent heating efficiency of SPIONs has been previously described, our objective was to probe the sensitivity limits of LIT. For certain size regimes it was possible to detect signals at concentrations as low as $0.1 \mathrm{mg} \mathrm{Fe} / \mathrm{mL}$. Measuring at different concentrations enabled a linear regression analysis and extrapolation of the limit of detection for different size nanoparticles.
\end{abstract}




\section{Introduction}

Superparamagnetic iron oxide nanoparticles (SPIONs) are fascinating materials for biomedical applications due to their biocompatibility [1-4] and capabilities of generating heat when exposed to an alternating magnetic field (AMF) [5]. These features, in combination with their nanoscale size and versatility, render them particularly intriguing over a wide range of disciplines, including drug delivery, magnetic resonance imaging (MRI) and hyperthermia treatment [6], the latter having been recently approved in Europe for the treatment of glioblastoma multiforme [7]. The success of these SPION-mediated hyperthermia treatments relies strongly on the local concentration of SPIONs within the tumor and their heating potential in the region of interest. In turn, the latter factor is dictated by the specific material properties (e.g., size shape, crystallinity etc.) and external parameters, such as magnetic field strength and frequency [5, 8-14]. While field strength and frequency can be easily controlled, a myriad of variables affect the physico-chemical makeup of SPIONs and consequently their overall ability to generate heat. As an example, it has been reported that significant variations in the heating power also occur in SPIONs produced on an industrial scale [15]. As a result, experimentally assessing heating power in every synthetic batch is highly recommended as a means of quality control. This work presents a comparative screening of the thermal SPION characteristics via LIT as an extension to Monnier et al. [16]. The lower detection limit of the LIT system as a function of different particle diameters was investigated by measuring thermal properties at decreasing concentrations.

There are numerous ways to measure and quantify the heating potential of a respective SPION sample. Standard calorimetric methods are arguably the most common ones, which rely on monitoring the transient temperature change (e.g. via fiber optic cables) of the sample over time after exposure to an AMF $[6,17]$. The heating slope ( $\Delta$ Temperature/ $\Delta$ time) within the initial seconds of exposure can then be deduced $[6,8,18]$. However, inconsistencies in sample measurement and difficulty interpreting these data are becoming increasingly recognized, and call into question how reliable these approaches are and to what extent the results can be reproduced $[17,18]$. A very promising contactless alternative method is lock-in thermography (LIT). Pioneered in 1984 [19], this method assumes that the stimulus (i.e., the AMF), and consequently the heat dissipating from the excited material (i.e., the magnetic nanoparticles) are periodically modulated [20]. In this particular case, the result of LIT demodulation would be an amplitude image showing within the AMF the heating signature which originates from only the magnetic nanoparticles (MNPs). 
Here, MNP batches of different sizes prepared within the same research group were synthesized by thermal decomposition, coated with citric acid and transferred to an aqueous environment. The MNP in the measurements were varied only by size and concentration. A multi-sample holder enabled screening and comparison of multiple samples/parameters simultaneously. A colloidal suspension of commercially available nanoparticles was also included in each experiment to act both as a guide value within the measurement series (i.e., to assure that values or conditions did not vary between them) and as an overall reference standard independent of the research group.

\section{Experimental section}

\subsection{Synthesis of SPIONs and ligand exchange}

All chemicals were used as received without any further purification. Each aqueous solution was prepared with deionized water received from a Milli-Q system (resistivity $=18.2 \mathrm{M} \Omega \mathrm{cm}$, Millipore AG). The SPIONs used in this work were prepared via thermal decomposition of an iron oleate complex in the presence of oleic acid [21, 22]. The desired nanoparticle sizes could be varied by changes in the defined heating ramps and by minor changes to the iron (III) chloride hexahydrate to sodium oleate ratio, and iron oleate complex proportion to oleic acid. To stabilize the SPIONs in aqueous solutions a ligand exchange with citric acid (CA) was performed [23].

\subsection{Transmission electron microscopy}

Transmission electron microscopy (TEM) was used to determine size, dispersity and morphology of the synthesized SPIONs. Sample preparation was performed by following a method described by Michen and Geers, et al. [24]. TEM experiments were carried out on a FEI Tecnai Spirit operating at a voltage of $120 \mathrm{kV}$ and equipped with a side-mounted Veleta camera. Particle size analysis was performed using ImageJ (v1.46r) particle sizing software (n > 1500 nanoparticles).

\subsection{Dynamic light scattering}

Dynamic light scattering (DLS) measurements were performed at $25{ }^{\circ} \mathrm{C}$ using a commercial goniometer instrument (3D LS Spectrometer, LS Instruments AG, Switzerland). Three measurements were made at a scatterings angle $90^{\circ}$ and autocorrelation functions were analyzed with a customized script in Mathematica (Version 10.1, Wolfram Research Inc). 


\subsection{Iron quantification by ICP-OES}

Iron quantification of SPIONs was achieved through Inductively Coupled Plasma Optical Emission Spectroscopy (ICP-OES, PerkinElmer Optima 7000 DV). A triplet of each nanoparticle suspension $(10 \mu \mathrm{L})$ was dissolved in aqua regia $\left(\mathrm{HNO}_{3}: \mathrm{HCl}\right.$ with a volume ratio of $1: 3,500 \mu \mathrm{L}$ per tube), transferred to $15 \mathrm{~mL}$ Falcon tubes (BD Biosciences, Switzerland) and diluted to $10 \mathrm{~mL}$ with Milli-Q water. Additionally a standard curve of aqueous iron solution was prepared and all samples were measured by ICP-OES at a wavelength of $238.2 \mathrm{~nm}$ for iron.

\subsection{Vibrating sample magnetometer}

Magnetic properties of the citric acid coated SPIONs were determined by a vibrating sample magnetometer (VSM, Model 3900, Princeton Measurements Corporation). Nanoparticle suspensions were deposited onto hydrophobic cotton pellets and dried overnight. The samples were attached to the sample holder with grease and hysteresis loop measurements were performed at room temperature using a measurement averaging time of $300 \mathrm{~ms}$, and a constant field increment. The resulting magnetization curves were normalized by the dried sample mass of iron.

\subsection{Thermal measurements}

All thermal measurements were performed with a previously described lock-in thermal imaging setup [16]. SPIONs were exposed to an alternating magnetic field generated by a commercial coil system (Magnetherm ${ }^{\mathrm{TM}} \mathrm{V} 1.5$, nanoTherics Ltd) consisting of a water cooled coil, a laboratory power supply (EA-PS 3032-20B, EA Elektro Automatic) and a function generator (SFG-2004, GW Instek). The apparatus was set to run at $524.5 \mathrm{kHz}$ and $18.5 \mathrm{mT}$. The heating rates were determined using an infrared camera (Onca-MWIR-InSb-320, XenICs) mounted on a standard microscope stand (Leica Microsystems), and recorded infrared images with a full frame rate of $250 \mathrm{~Hz}$. Data were transferred to a personal computer and processed in real time. The modulation frequency was set to $1 \mathrm{~Hz}$ for all measurements. LIT measurements were performed in 4 chambers of a nine-well plate. It was verified that the magnetic field was comparable in the 4 positions used for measurement, and characterization of the magnetic field is included in a forthcoming manuscript [25].

\section{Results and discussion}



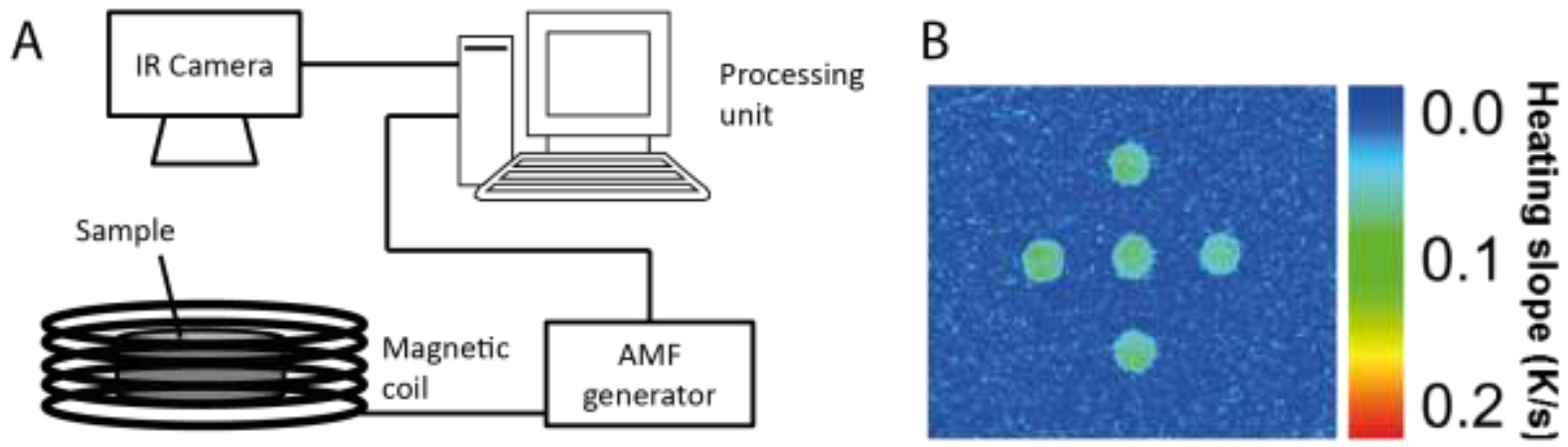

Figure 1. A simplified scheme of the lock-in thermography system (A) consisting of the sample, a magnetic coil, a thermal imaging device and processing unit. Under implementation of a customized lock-in demodulation algorithm, the output is a two-dimensional image in which every pixel can be related to the initial heating slope values (B).

A typical LIT setup is composed of three main components (Figure 1A): an infrared camera to record the thermal emission of the sample under investigation, a stimulation scheme to periodically excite the sample under test, and a processing unit to demodulate the transient infrared images recorded by the camera. The infrared camera captures the temperature variations of the sample in the form of an infrared image stack that is demodulated by the processing unit. The main advantage of LIT lies in its ability to filter out any low-varying spurious signals not originating from the nanoparticles (such as heat originating from the coil itself). In addition, the method is very precise, and many modulation cycles can be averaged to reach the desired sensitivity. The output of the LIT demodulation is a 2D image whereby every pixel can be related to the initial heating slope value. While the heating efficiency and its dependence on nanoparticle size [16, 26-28] has been described on multiple occasions, the aim of this study was to investigate the minimum limit of detection as a factor of nanoparticle size and concentration. 

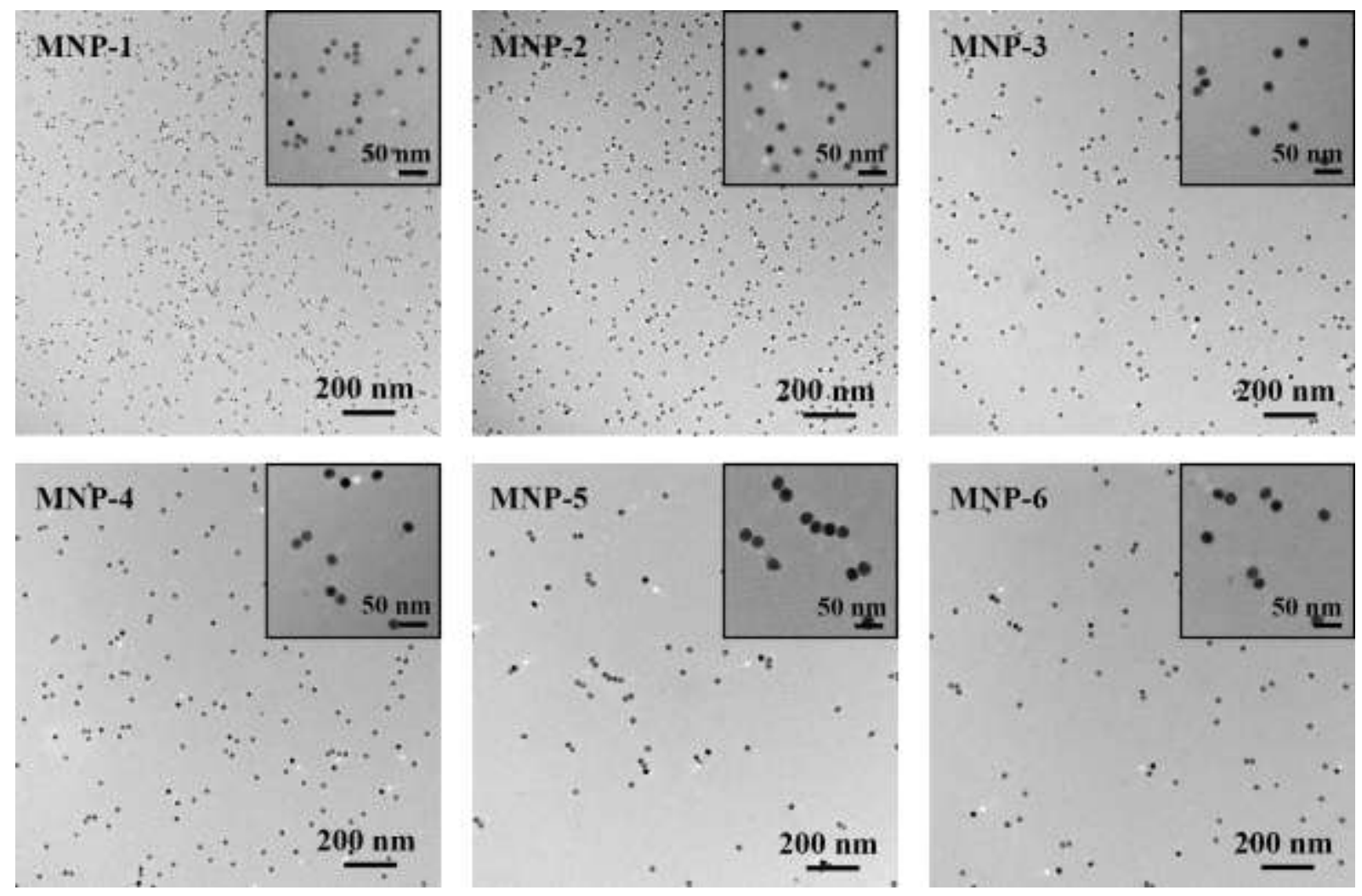

Figure 2. TEM micrographs of CA coated SPIONs, all synthesized by thermal decomposition and with mean size distributions between 13 and $25 \mathrm{~nm}$.

All SPIONs used in this trial were synthesized by thermal decomposition, and a ligand exchange was performed to coat them with citric acid, yielding colloidally stable MNPs in water [21-23]. SPION size was varied by making minor adjustments to precursor concentrations in the reaction. Figure 2 shows TEM images of the different SPIONS, and core diameters were found to be between approximately 13 and $25 \mathrm{~nm}$ (Figure 3A, C). MNP colloidal stability and size was further affirmed by DLS (Figure 3B). All particles were found to be colloidally stable in $\mathrm{H}_{2} \mathrm{O}$, and hydrodynamic diameters $\left(\mathrm{d}_{H}\right)$ were found to between 14 and $25 \mathrm{~nm}$ (Fig. 3B-C), matching closely with core diameters measured via TEM and confirming that they were monodisperse in water. MNP-5 exhibits a broader size distribution due to variances in the synthesis. As demonstrated by Hufschmid et al. [29] adding oleic acid or reducing iron concentration broadens the size distribution. Since the mean size is close to MNP-6 it was interesting to investigate in which way a broader dispersity influences the heating slope of the materials. Magnetization as a function of magnetic field strength was measured by a vibrating sample magnetometer. Magnetization was normalized by mass of iron, and Figure 3D shows magnetization curves for each size SPION with no signs of 
hysteresis or coercivity exhibited by any samples, therefore indicating superparamagnetic behavior.

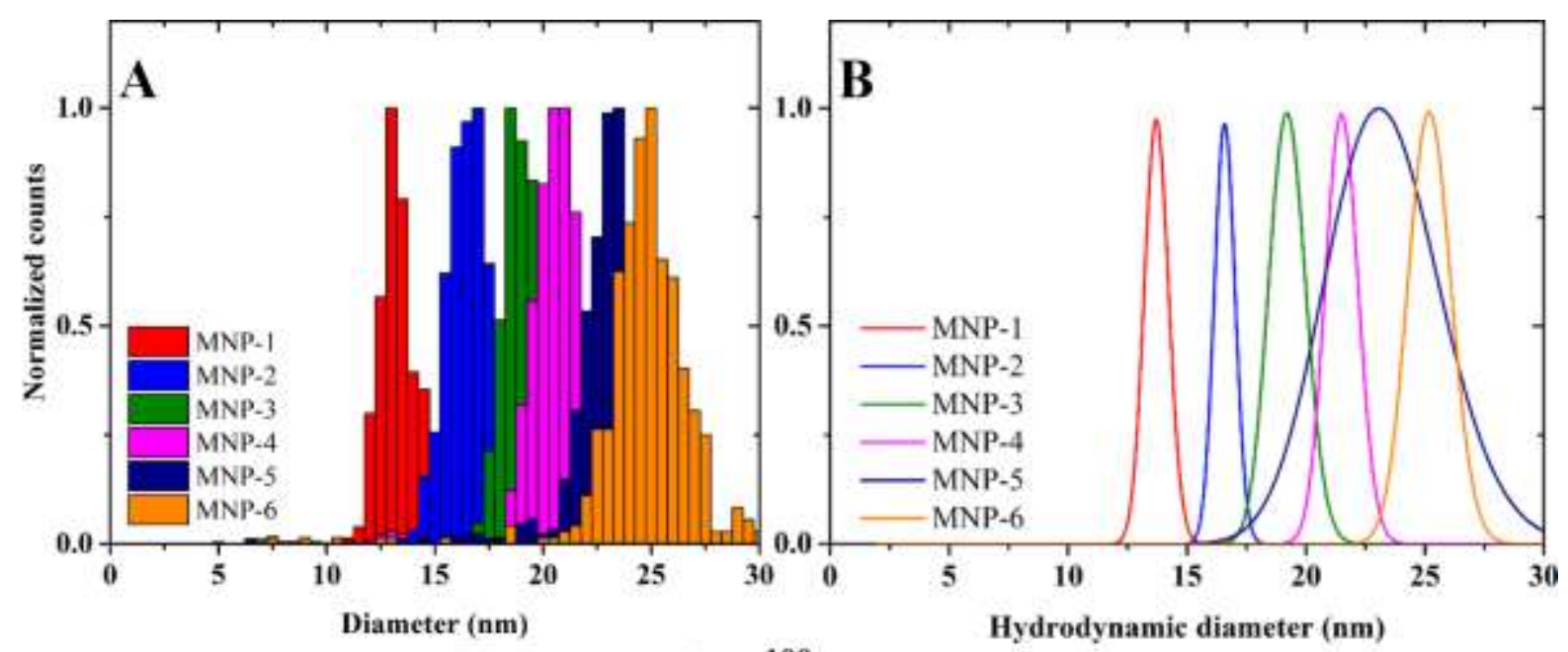

\begin{tabular}{ccc}
\hline $\mathbf{C}$ & $\mathbf{d}_{\text {TEM }}(\mathbf{n m})$ & $\mathbf{d}_{\boldsymbol{H}, \mathrm{DLS}}(\mathbf{n m})$ \\
\hline MNP-1 & $13 \pm 1$ & $14 \pm 3$ \\
MNP-2 & $16 \pm 2$ & $17 \pm 3$ \\
MNP-3 & $19 \pm 3$ & $19 \pm 4$ \\
MNP-4 & $21 \pm 4$ & $22 \pm 4$ \\
MNP-5 & $24 \pm 6$ & $24 \pm 8$ \\
MNP-6 & $25 \pm 2$ & $25 \pm 5$ \\
\hline
\end{tabular}

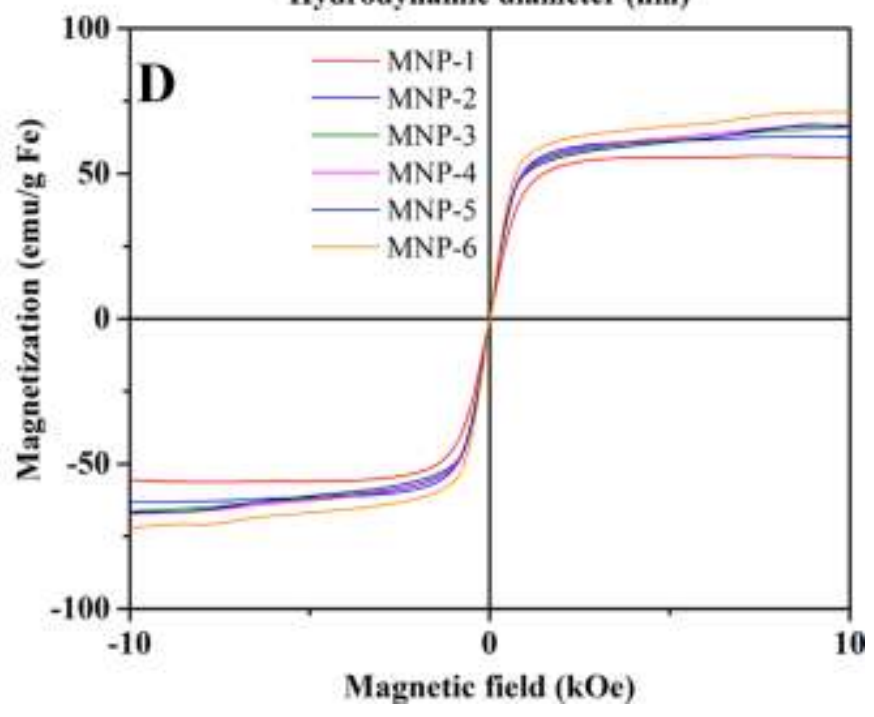

Figure 3. Size and magnetic characterization of MNPs. Size histograms were determined by TEM micrographs (A). Nanoparticle stability and hydrodynamic diameter $\left(\mathrm{d}_{H}\right)$ were determined via DLS (B). A summary table shows the core diameter measured by TEM and $\mathrm{d}_{H}$ measured by DLS (C). Sizes represent mean \pm SD. Vibrating sample magnetometry highlighted the superparamagnetic nature of the MNPs (D).

These respective SPIONs were subsequently investigated by LIT. Every measurement array consisted of one batch diluted to four different concentrations, as well as one central reference consisting of a suspension of commercially available batch, (Chemicell fluidMAG-D, Fig. 4). This sample was diluted to $0.5 \mathrm{mg} \mathrm{Fe} / \mathrm{mL}$ and included to assure continuity between the individual measurements. The signal generated by the reference sample was highly reproducible, and yielded an average heating slope of $8.6 \pm 0.2 \mathrm{mK} / \mathrm{s}$ in every measurement. Furthermore, it provides a broader, commercially accessible reference for the community. 


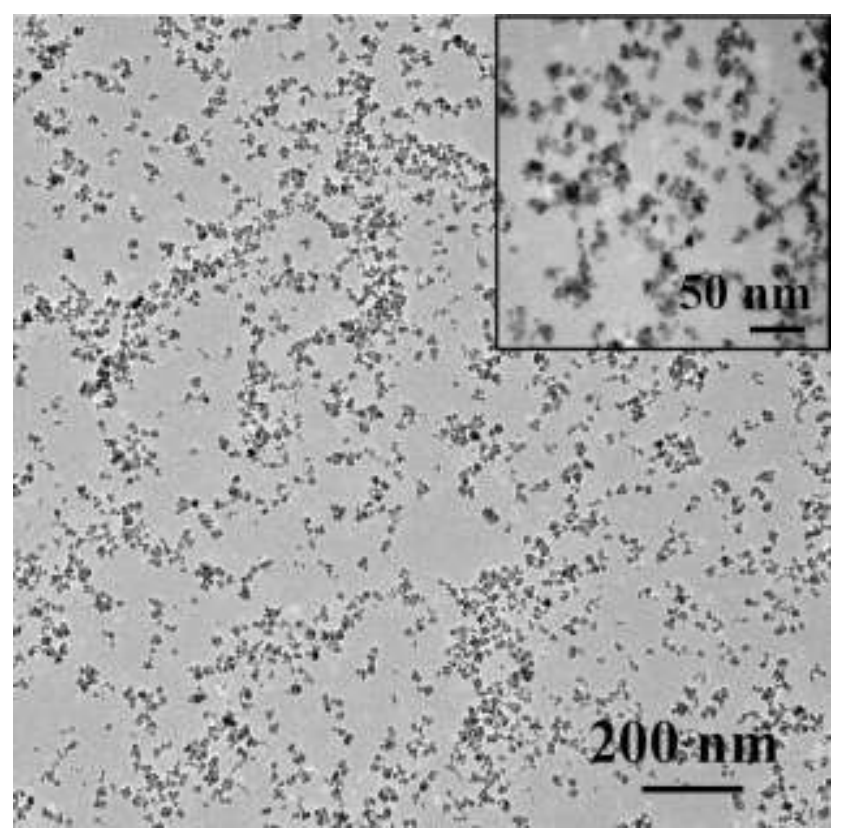

Figure 4. TEM images of commercial Chemicell fluidMAG-D SPIONs.

The experimental setup, described above, is schematically represented in Figure 5A. MNP samples of individual sizes were diluted to varying concentrations (e.g. 0.1 to $1.0 \mathrm{mg}$ $\mathrm{Fe} / \mathrm{mL}$ ) and the output data from LIT is processed and displayed as a 2D image where each pixel represents heating slope values that can be averaged over the sample area. The overall heating originating from the MNPs (dispersed in Milli-Q) with concentrations ranging from 0.2 to $4.0 \mathrm{mg} \mathrm{Fe} / \mathrm{mL}$ and stimulated by an AMF were measured via LIT, resulting in at least two detectable signals for each particle size at higher concentrations of 1 and $4 \mathrm{mg} \mathrm{Fe} / \mathrm{mL}$ (Fig. 5B). Varying the concentrations of the MNP suspension lead to a change of the heating slope, and extrapolation of these values showed that the impact of the particle size increased with higher concentrations. However, to investigate the sensitivity of the system, a second measurement series was prepared in a concentration range from 0.1 to $1.0 \mathrm{mg} \mathrm{Fe} / \mathrm{mL}$. Measurement of the smaller MNP samples (i.e. MNP-1, MNP-2, and MNP-3) were intentionally avoided as the initial experiments already approached the limit of detection, defined as the baseline average signal of the solvent in which the measurement was taken, for these very small MNP. For the MNP-4, MNP-5, and MNP-6, i.e. the larger nanoparticles, it was possible to measure concentrations down to approximately $0.1 \mathrm{mg} \mathrm{Fe} / \mathrm{mL}$ in a second set of experiments (Fig. 5C). The heating slope value for MNP-4 at the lowest concentration of $0.1 \mathrm{mg} \mathrm{Fe} / \mathrm{mL}$ (i.e., $0.98 \mathrm{mK} / \mathrm{s}$ ) was only minimally above the background signal (i.e., $0.87 \mathrm{mK} / \mathrm{s}$ ), however for MNP-5 and MNP-6 at the same concentration significantly exceeded this lower limitation (1.78 and $2.73 \mathrm{mK} / \mathrm{s}$, respectively). 

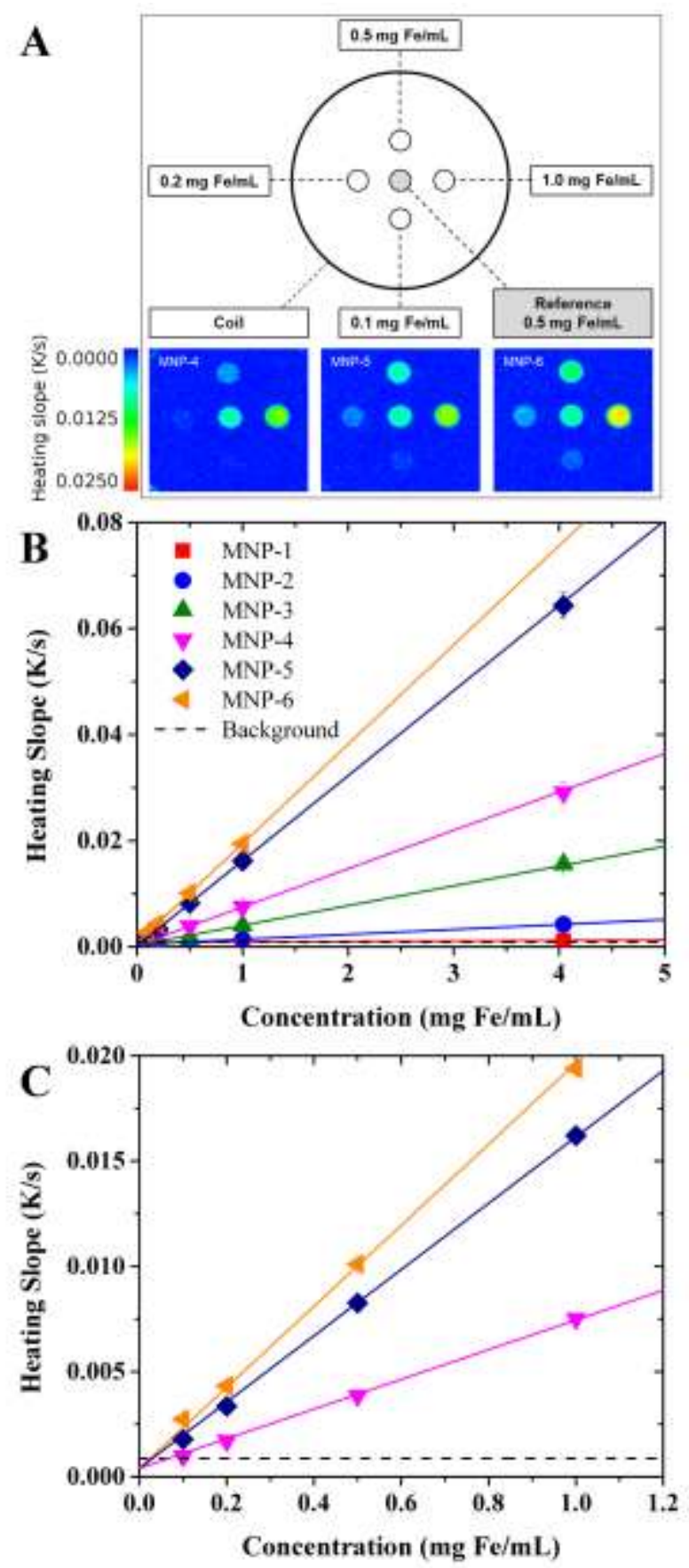

Figure 5. Investigation of different sized SPIONs and their thermal signals by LIT. Schematic representation of LIT experimental setup and representative results from LIT measurements (A). Measurements of heating slope as a function of MNP concentration for various sizes as measured by LIT (B). Data points represent triplicate experiments, and solid lines show best fit linear regression curves through the data. Second experiments at lower concentrations, approaching the limit of detection, for MNP-4, MNP-5, and MNP-6 (C).

The detectable limit for concentrations of MNP-4, MNP-5, and MNP-6 were predicted to be $0.07,0.03$, and $0.02 \mathrm{mg} \mathrm{Fe} / \mathrm{mL}$, respectively, by reference to the extrapolation. This simulated 
result was consistent with our measurements, which displayed that LIT can be used at very low concentrations depending on the heating properties of the particles.

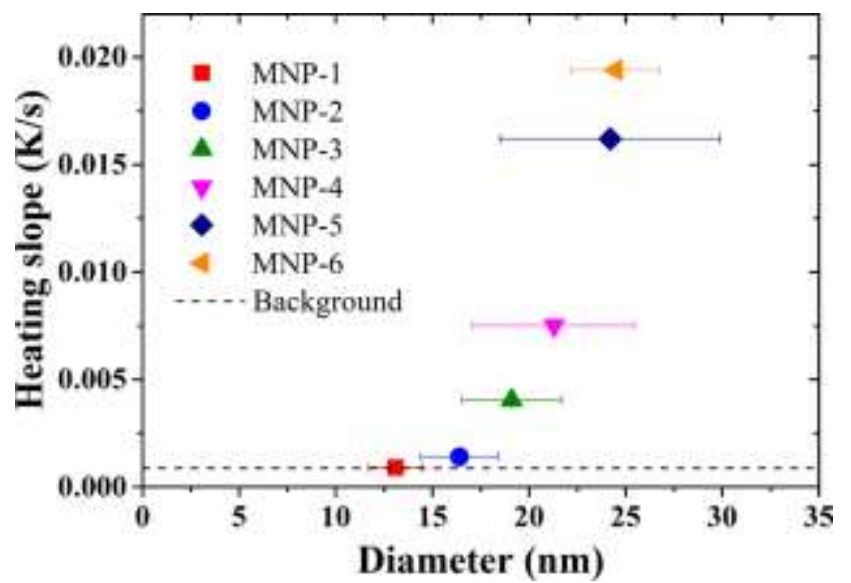

Figure 6. LIT measurement of MNP heating slope at $1 \mathrm{mg} \mathrm{Fe} / \mathrm{mL}$ as a function of nanoparticle diameter.

Based on this data, it was possible to evaluate heating slopes as a function of the nanoparticle diameters at a concentration of $1 \mathrm{mg} \mathrm{Fe} / \mathrm{mL}$ (Fig. 6). The heating slope strongly depends on the diameter of the particles, as has been previously described $[8,26,30]$. The standard deviation of these measurements (in the range of $0.02 \mathrm{mK} / \mathrm{s}$ to $0.2 \mathrm{mK} / \mathrm{s}$ ) confirmed the outstanding comparability of the result. These data emphasize that the LIT technique is reliable and continuous, and that single measurements can be compiled without further alignments or variables.

\section{Conclusion}

The potential of LIT as a characterization method for thermally active nanoparticle was demonstrated by examining the heating potential of monodisperse, colloidally stable SPIONs. Batches of SPIONs of increasing size, as well as a commercially available SPION formulation, were tested for heating at different concentrations. The commercially available sample was kept at a constant concentration throughout each measurement, and the measured heating potentially was found to be highly consistent and reproducible. This indicates the stability of LIT as a characterization technique that requires minimal post-processing and manipulation. Furthermore, measurement of heating potential at different concentrations showed a linear relationship between particle concentration and heating potential, specific to particle characteristics (e.g. size). It was therefore possible to extrapolate this relationship, and estimated limits of detection were closely approximated by experimental data. In the context 
of using SPIONs for biomedical applications (in particular for hyperthermia treatment), thermal characterization is of utmost importance, as AMF-induced SPION heating is influenced by a myriad of factors. Thus, characterizing the heating potential is critical for understanding the efficacy of each individual particle batch. LIT was found to be highly sensitive, able to detect certain sizes of nanoparticle down to $0.1 \mathrm{mg} \mathrm{Fe} / \mathrm{mL}$, and therefore provides an efficient, objective, and reproducible approach to screen batches and compare their thermal properties.

\section{Acknowledgements}

The authors gratefully acknowledge the financial support from the Swiss National Science Foundation (159803), the University of Fribourg and the Adolphe Merkle Foundation. Parts of this work were supported by the National Center of Competence in Research Bio-Inspired Materials. Access to VSM was kindly provided by the Institute for Geophysics of the ETH Zurich. The authors thank Dr. Sandor Balog for the Mathematica script to analyze DLS data.

\section{References}

[1] A.R. Jalilian, A. Panahifar, M. Mahmoudi, M. Akhlaghi, and A. Simchi, "Preparation and biological evaluation of $\left[{ }^{67} \mathrm{Ga}\right]$-labeled-superparamagnetic nanoparticles in normal rats," Radiochim. Acta, vol. 97, pp. 51-56, 2009.

[2] B. Polyak and G. Friedman, "Magnetic targeting for site-specific drug delivery: applications and clinical potential," Expert Opin. Drug Deliv., vol. 6, no. 1, pp. 53-70, 2009.

[3] C.H. Cunningham, T. Arai, P. C. Yang, M.V. McConnell, J.M. Pauly, and S.M. Conolly, "Positive Contrast Magnetic Resonance Imaging of Cells Labeled with Magnetic Nanoparticles,” Magn. Reson. Med., vol. 53, no. 5, pp. 999-1005, 2005.

[4] S. Saini, R. Sharma, R.L. Baron, D.A. Turner, P.R. Ros, P.F. Hahn, W.C. Small, E.E. de Lange, A.E. Stillman, R.R. Edelman, V.M. Runge, and E.K. Outwater, "Multicentre Dose-Ranging Study on the Efficacy of USPIO Ferumoxtran-10 for Liver MR Imaging," Clin. Radiol., vol. 55, pp. 690-695, 2000.

[5] R.E.E. Rosensweig, "Heating magnetic fluid with alternating magnetic field," J. Magn. Magn. Mater., vol. 252, pp. 370-374, 2002.

[6] S. Laurent, S. Dutz, U.O. Häfeli, and M. Mahmoudi, "Magnetic fluid hyperthermia : Focus on superparamagnetic iron oxide nanoparticles," Adv. Colloid Interface Sci., vol. 166, pp. 8-23, 2011. 
[7] K. Maier-Hauff, F. Ulrich, D. Nestler, H. Niehoff, P. Wust, B. Thiesen, H. Orawa, V. Budach, and A. Jordan, "Efficacy and safety of intratumoral thermotherapy using magnetic iron-oxide nanoparticles combined with external beam radiotherapy on patients with recurrent glioblastoma multiforme," J. Neurooncol., vol. 103, no. 2, pp. 317-324, 2011.

[8] A.E. Deatsch and B.A. Evans, "Heating efficiency in magnetic nanoparticle hyperthermia," J. Magn. Magn. Mater., vol. 354, pp. 163-172, 2014.

[9] R. Hergt, S. Dutz, R. Müller, and M. Zeisberger, "Magnetic particle hyperthermia: nanoparticle magnetism and materials development for cancer therapy," J. Phys. Condens. Matter, vol. 18, no. 38, pp. S2919-S2934, 2006.

[10] C.S.S.R. Kumar and F. Mohammad, "Magnetic nanomaterials for hyperthermia-based therapy and controlled drug delivery," Adv. Drug Deliv. Rev., vol. 63, pp. 789-808, 2011.

[11] D.L. Leslie-Pelecky and R.D. Rieke, "Magnetic Properties of Nanostructured Materials," Chem. Mater., vol. 8, pp. 1770-1783, 1996.

[12] W.J. Atkinson, I.A. Brezovich, and D.E.V.P. Chakraborty, "Usable Frequencies in Hyperthermia with Thermal Seeds," IEEE Trans. Biomed. Eng., vol. BME-31, no. 1, pp. 70-75, 1984.

[13] A.K. Gupta and M. Gupta, "Synthesis and surface engineering of iron oxide nanoparticles for biomedical applications," Biomaterials, vol. 26, no. 18, pp. 39954021, 2005.

[14] B. Kozissnik, A.C. Bohorquez, J. Dobson, C. Rinaldi, B. Kozissnik, A.C. Bohorquez, J. Dobson, and C. Rinaldi, "Magnetic fluid hyperthermia: Advances , challenges , and opportunity," Int. J. Hyperth., vol. 29, no. 8, pp. 706-714, 2013.

[15] M. Kallumadil, M. Tada, T. Nakagawa, M. Abe, P. Southern, and Q.A. Pankhurst, "Suitability of commercial colloids for magnetic hyperthermia," J. Magn. Magn. Mater., vol. 321, pp. 1509-1513, 2009.

[16] C.A. Monnier, M. Lattuada, D. Burnand, F, Crippa, J. Martinez-Garcia, A. Hirt, B. Rothen-Rutishauser, M. Bonmarin, A. Fink, "A lock-in-based method to look through the thermal signatures of magnetic nanoparticles in liquid, solid and aggregated states," Nanoscale, vol. 8, pp. 13321-13332, 2016.

[17] R.R. Wildeboer, P. Southern, and Q.A. Pankhurst, "On the reliable measurement of specific absorption rates and intrinsic loss parameters in magnetic hyperthermia materials," J. Phys. D. Appl. Phys., vol. 47, pp. 495003-495017, 2014. 
[18] I. Andreu, E. Natividad, "Accuracy of available methods for quantifying the heat power generation of nanoparticles for magnetic hyperthermia generation of nanoparticles for magnetic hyperthermia," Int. J. Hyperth., vol. 29, no. 8, pp. 739-751, 2013.

[19] O. Breitenstein, W. Warta, and M. Langenkamp, Lock-in thermography: Basics and use for evaluating electronic devices and materials. Springer, Heidelberg, 2010.

[20] T. Ahmed, H.J. Jin, X. Wang, L.D. Favro, P.K. Kuo, and R.L. Thomas "Infrared Thermal Wave Studies of Composites," in: D.O. Thompson and D.E. Chimenti (Eds.), Rev. Prog. Quant. Nondestruct. Eval., vol. 10B, Springer, New York, pp. 2173-2179, 1991.

[21] J. Park, K. An, Y. Hwang, J-G. Park, H-J. Noh, J-Y. Kim, J-H. Park, N-M. Hwang, and T. Hyeon "Ultra-large scale syntheses of monodisperse nanocrystals via a simple and inexpensive route," Nature, vol. 3, no. 12, pp. 1-13, 2004.

[22] S. Sun and H. Zeng, "Size-Controlled Synthesis of Magnetite Nanoparticles," J. Am. Chem. Soc., vol. 124, no. 31, pp. 8204-8205, 2002.

[23] M. Lattuada and T.A. Hatton, "Functionalization of Monodisperse Magnetic Nanoparticles," Langmuir, vol. 23, no. 4, pp. 2158-2168, 2007.

[24] B. Michen, C. Geers, D. Vanhecke, C. Endes, B. Rothen-Rutishauser, S. Balog, A. Petri-Fink, "Avoiding drying-artifacts in transmission electron microscopy: Characterizing the size and colloidal state of nanoparticles," Sci. Rep., vol. 5, p. 9793 , 2015.

[25] C.A. Monnier, F. Crippa, C. Geers, M. Lattuada, M. Bonmarin, "Lock-in thermography as a tool for magnetic nanoparticles: New Insights on intrinsic loss of power values and magnetic fields," under preparation.

[26] M. Gonzales-Weimuller, M. Zeisberger, and K.M. Krishnan, “Size-dependant heating rates of iron oxide nanoparticles for magnetic fluid hyperthermia," J. Magn. Magn. Mater., vol. 321, no. 13, pp. 1947-1950, 2009.

[27] R. Levy, "Expectation-based syntactic comprehension," Cognition, vol. 106, pp. 11261177, 2008.

[28] J. Fortin, C. Wilhelm, J. Servais, C. Ménager, J. Bacri, and F. Gazeau, "Size-Sorted Anionic Iron Oxide Nanomagnets as Colloidal Mediators for Magnetic Hyperthermia," J. Am. Chem. Soc., vol. 129, no. 9, pp. 2628-2635, 2007.

[29] R. Hufschmid, H. Arami, R.M. Ferguson, M. Gonzales, E. Teeman, L.N. Brush, N.D. Browning, and K.M. Krishnan, "Synthesis of phase-pure and monodisperse iron oxide 
nanoparticles by thermal decomposition.," Nanoscale, vol. 7, no. 25, pp. 11142-54, 2015.

[30] M.A. Gonzalez-Fernandez, T.E. Torres, M. Andrés-Vergés, R. Costo, P. de la Presa, C. J. Serna, M.P. Morales, C. Marquina, M.R. Ibarra, and G.F. Goya, "Magnetic nanoparticles for power absorption: Optimizing size, shape and magnetic properties," $J$. Solid State Chem., vol. 182, no. 10, pp. 2779-2784, 2009. 